\title{
Usefulness of C-reactive Protein Testing in Acute Cough/Respiratory Tract Infection: A Case-control Study in a UK Based GP Population
}

\author{
Ghosh $\mathbf{S}^{1^{*}}$, Farell $\mathrm{L}^{2}$, Calnan $\mathbf{G}^{3}$ and Panray $\mathbf{G B}^{1}$ \\ ${ }^{1}$ De-Montfort University, Leicester UK, Enderby Medical Centre, Leicestershire, UK \\ ${ }^{2}$ Enderby Medical Centre, Leicestershire, UK \\ ${ }^{3}$ POC testing Limited, Angus UK
}

*Corresponding author: Ghosh S, GP, Faculty of Health Sciences, De-Montfort University, Leicester UK, Enderby Medical Centre, Leicestershire, UK, Tel: 01162078857; E-mail:sudip.ghosh@dmu.ac.uk

Received date: April 27, 2017; Accepted date: April 29, 2017; Published date: April 29, 2017

Copyright: (C) 2017 Ghosh S, et al. This is an open-access article distributed under the terms of the Creative Commons Attribution License, which permits unrestricted use, distribution, and reproduction in any medium, provided the original author and source are credited.

\begin{abstract}
Introduction: Point of care testing for C-reactive protein (CRP) has shown promise as a measure to reduce unnecessary antibiotic prescribing in respiratory tract infections (RTI) and has been adopted into NICE guidelines, but its use in primary care is still controversial. We aimed to evaluate the effect of CRP testing on the prescription of antibiotics and the outcome of patients in general practice with acute cough/RTI.
\end{abstract}

Methods: We undertook a case-control study with POC testing with CRP in intervention group (Oct 16-Jan 17) and compared this to similar cohort of patients from a year previously coded with exact diagnosis.

Results: A total of 207 patients were included: 69 in the intervention group and 138 in the control group.

The two groups were similar in clinical characteristics. In the intervention group, the antibiotic prescribing rate was $31.6 \%$, which was significantly lower than that in the control group $(59.9 \%)(P=0.003)$.

The recovery rate, as recorded by the GPs, was $94.9 \%$ and $93.8 \%$ in the intervention and control groups, respectively. Referral to Emergency Department for children under 12 was also reduced significantly in the intervention group (4.4\%) when compared to the control group $(6.5 \%$; $P=0.00479)$.

Conclusion: The study showed that CRP testing in patients with acute cough/RTI may reduce antibiotic prescribing and referral to pediatric ED, probably without compromising recovery.

Keywords: General Practitioners; Point-of-care tests; Antibiotic prescriptions; Respiratory tract Infection

\section{Introduction}

Primary care accounts for $80-90 \%$ of antibiotic prescriptions [1-3] most of which are for acute respiratory tract infections (RTIs) which in turn is one of the commonest reasons for patients to see their General Practitioners (GP) [4-6]. As an aid in determining infection severity, physicians sometimes use C-reactive protein (CRP) point-of-care tests (POCT).

Studies have highlighted that patient elements such as anxiety, lack of understanding about antibiotics, confidence in the health professional and physician factors such as patient-parent pressure, meeting expectations, complaints, litigations and time constraints further encouraged inappropriate antibiotic prescribing [7-9].

Difficulty in distinguishing between viral and bacterial respiratory tract infections has been recognized and has been widely debated. The value of CRP testing to distinguish between the two aetiologies has also been widely investigated. Butler et al concluded that clinical presentations could not explain the wide variation in antibiotic prescribing in primary care in 13 European countries [6].
The development of POCT provides an almost immediate diagnostic test which can impact a GP's antibiotic prescribing and thus aiding clinical acumen [10].

CRP is an acute phase biological marker which increases rapidly during infections more so in bacterial than in viral but also in inflammation and tissue injury [11]. A systematic review and metaanalysis of 13 studies showed that POC CRP testing was associated with a decrease in antibiotic prescribing [12]. A more recent Cochcrane database review also concluded a reduction in antibiotic use in RTIs in primary care and had no impact on patient recovery or duration of illness [13].

The review did however note a possible chance finding of increased hospitalization in one study by Little and co-investigators [14].

The House of Commons Health Committee has acknowledged the unprecedented pressures the NHS is facing with more admissions to hospitals especially during the winter months [15]. By adopting the guidelines from the National Institute of Health and Care Excellence (NICE) [16-17]; our study aims to evaluate the effect of CRP testing on 
Page 2 of 4

the prescription of antibiotics and the outcome of patients in a UK general practice cohort of patients presenting with acute cough/RTI.

\section{Methods}

\section{Design}

This was an evaluation study of patients presenting with symptoms of a respiratory tract infection (RTI) in a General Practice (GP) surgery between November 2016 to January 2017. This was then compared to a control group for the similar period ( 3 months) a year previously in a 1: 2 ratio.

\section{Patient selection}

In the intervention group, patients underwent a clinical assessment by the GP or Nurse Practitioner, who used a clinical scoring system similar to one used by Van-Vugt et al. [18] in the Netherlands (Table $1)$.

\begin{tabular}{|l|l|l|l|}
\hline & Intervention group (N= 69) & Control Group (N=138) & P value \\
\hline Age, mean (range) & $29.6(4-71)$ & $30.9(5-73)$ & 0.0734 \\
\hline Gender (M/F, \%) & $57 / 43$ & $53 / 47$ & 0.0893 \\
\hline Co-morbidities & & & \\
\hline Diabetes & 2 & 9 & 0.769 \\
\hline COPD & 0 & 1 & 0.129 \\
\hline IHD & 11 & 17 & 0.117 \\
\hline
\end{tabular}

Table 1: Baseline Characteristics of the 207 patients.

The control group was identified from patients seen in the exact time frame a year previously and identified from read codes used for diagnosis. These read codes included acute sinusitis, otitis media, tonsillitis, bronchitis and lower respiratory tract infection.

The criteria for inclusion and exclusion of patients were as identical as possible in both groups of the study. Patients were aged between 4 years to 75 years, suffered from symptoms of acute RTI according to the clinical diagnosis of the health professional and provided verbal consent to the blood test.

Criteria for exclusion included: treatment with antibiotics in the previous 2 weeks, chronic liver disease, and major surgery in the preceding month, auto-immune and systemic disorders, renal replacement therapy and other inflammatory diseases.

No ethical approval was required for the study as this was part of a normal service evaluation program initiated by the Practice.

CRP levels were measured at the point of care testing (POCT) using the Spinit analyser (Biosurfit, Lisbon Portugal; POCT Limited is the UK distributer). Spinit is a point of care system for the rapid quantitative measurement of a number of different blood parameters, using Spinit disposable discs. The Spinit -CRP disposable disc is used to determine the $\mathrm{C}$-reactive protein concentration from a small capillary blood sample $(5 \mu \mathrm{L})$ in less than 6 minutes.

The microfluidic disc allows for automated sample processing and conducting the assay based on antibody-antigen reaction. The CRP measurement is performed in real time by the Spinit instrument using an optical-based detection system-Surface Plasmon Resonance (SPR). The Spinit-CRP disc is ready-to-use and is standardized against the ERM $^{\circ}$-DA474 from IFCC (International Federation of Clinical Chemistry).

Decision to prescribe antibiotics was made based on the CRP level and the presenting clinical criteria (Figure 1).

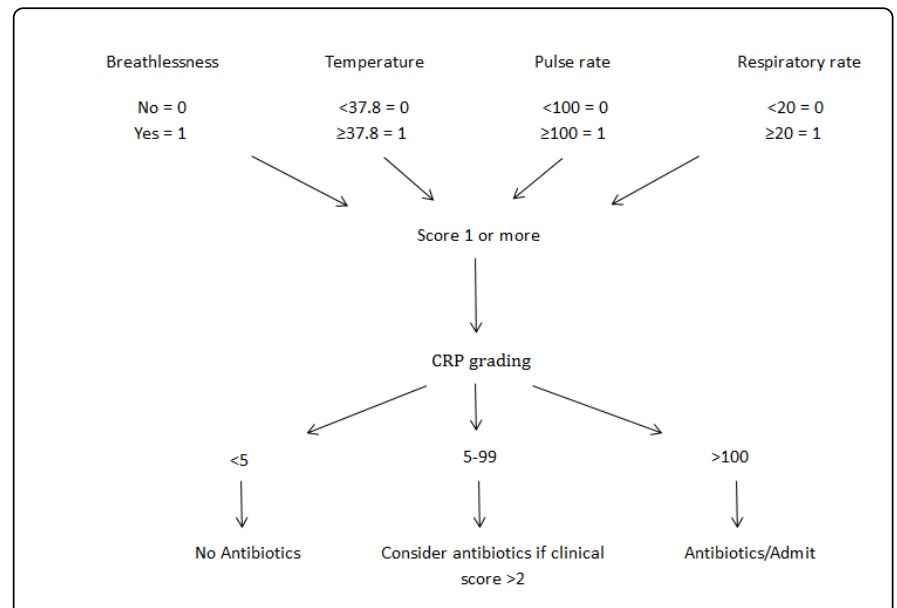

Figure 1: Clinical Criteria for Point of Care CRP testing.

Patients who did not receive antibiotics were given reassurance and advice. Data was also collected on referral to the emergency department (ED). This data was then compared to control group for analysis. Patients were followed up for 2 weeks post consultation in the intervention group and all control group patients had their notes analyzed for 2 further weeks post their initial consultation and reviewed against any emergency department or Out of Hours communications in the same time period.

\section{Statistical analysis}

For the outcomes used in the analysis of antibiotic usage and referrals to ED, the non-parametric Kruskal-Wallis test (continuous variables) and Pearson's Chi-squared test with simulated p-values (categorical variables) were applied. POCT guided therapy was regarded as non-inferior to standard therapy if no significant increase 
was observed. As all patients who received POCT was entered into analysis, the alpha $(\alpha)$ error was assumed at $5 \%$ and the study was powered to $80 \%$ to detect a $10 \%$ change in prescribing. The sample calculation required for a control group to be a minimum of 1.5 times that of intervention group and we settled for a ratio of 2:1 in this study. R version 2.5.1 (R Foundation for Statistical Computing, Vienna, Austria) and SPSS and SPSS 16.0 (SPSS, Inc., Chicago, IL, USA) were used for all analyses.

\section{Results}

A total of 207 patients were analyzed. The baseline characteristics are highlighted in Table 1. There was no statistical difference in patients treated in the interventional group compared to the control group. The mean age in the CRP tested group was 29.6 (SD 17.9) and 30.9 (SD 21.6) in the control group. No patient was older than 73 years in the analysis. Children aged 4-12 years made up 37 of the 69 patients (54\%) in the intervention group. There were no meaningful differences seen between the groups when compared to gender and comorbidities.

The CRP tested group had strict clinical criteria for testing at point of care. Patients who presented with a clinical score of at least 1 went on to have CRP testing. Antibiotic prescriptions were then deemed necessary according to CRP stratification as described in (Figure 1).

Table 2 highlights the results of the 69 patients in terms of their CRP point of care testing, the effect of antibiotic prescribing and their disbursement and clinical recovery over the following 2 weeks. CRP levels of $0-5,5-99,>100 \mathrm{mg} / \mathrm{L}$ were observed in 38,25 and 6 patients, respectively. Antibiotics were prescribed to patients with higher CRP levels $(0 \%, 43 \%$ and $83 \%$ for CRP scores of $0-5,5-99$ and $>100 \mathrm{mg} / \mathrm{L}$ ) (Table 2).

\begin{tabular}{|l|l|l|l|l|l|}
\hline \multirow{2}{*}{ CRP $(\mathrm{mg} / \mathrm{L})$} & \multirow{2}{*}{ Number } & Clinical Score & Test score & \multirow{2}{*}{ Antibiotics given } & \multirow{2}{*}{ Returned within 2 weeks and given Abx } \\
\cline { 3 - 6 } & & $01-\mathrm{Feb}$ & $\geq 2$ & & \\
\hline $01-$ May & 38 & 38 & 0 & 0 & $2(5.3 \%)$ \\
\hline May-99 & 25 & 21 & 4 & $16(42.9 \%)$ & $0(0 \%)$ \\
\hline$\geq 100$ & 6 & 4 & 2 & $5(83 \%)$ & $0(0 \%)$ \\
\hline Total & 69 & 63 & 6 & $21(31 \%)$ & $2(3 \%)$ \\
\hline
\end{tabular}

Table 2: CRP levels and clinical scores of patients, the percentage who were prescribed antibiotics, and the number who returned within 2 weeks.

This represented $31.6 \%$ of the intervention group. A large proportion of patients (38/69) presented with symptoms had low levels of CRP and mild symptoms. Only a very small proportion of the intervention patients were admitted to the Emergency department $(n=3 ; 4.4 \%)$. All of these were children who were under 12 years of age. Two of these patients were in the mid-range CRP level and only 1 had CRP level $>100 \mathrm{mg} / \mathrm{L}$.

The control group had 83 patients (59.9\%) who were prescribed antibiotics in total over the time period that was analyzed. In the initial consultations, 71 patients were prescribed antibiotics and a further 12 were prescribed treatment upon return within 12 weeks. 29 patients (21.4\%) were admitted to ED in the control group. However, subcohort analysis of the under 12's in the control revealed only 9 patients $(6.5 \% ; p=0.0479)$ were admitted to the emergency department.

\section{Discussion}

General Practice is an essential part of primary care and remains the major gate-keeper for most patients within the United Kingdom (UK) health economy. This is invariably leading to greater workload and financial pressures. Respiratory tract infections are a common mode of presentation to primary care and the prescription of antibiotics is often generated for both clinical and patient satisfaction reasons $[8,9,19]$. The rate of growth of antibiotic prescriptions within primary care has been alarming over the last decade [20-21] and there is a concerted to reduce this in the next five years.

Our study adds to the scant research that POC CRP testing influences prescribing within the primary care setting. Antibiotic use was reduced compared to the control group, especially in patient who present with less clearly defined clinical parameters; where without
POC CRP it is likely that possible unreliable examination findings or perceived patient expectation may have resulted in a prescription. Furthermore there was no effect on recovery rates. POC CRP testing in under 12 year olds resulted in less referral to the Emergency department. The experience was also sighted as a better way of educating patients on value of antibiotic prescriptions for common ailments as shown by other investigators [19].

POC testing is widely used in a few European countries and has shown interestingly to be associated with lower systemic antibiotic consumption [19]. Our study demonstrates that when used alongside clinical guidelines and physical assessments; C-reactive protein testing can drive down inappropriate antibiotic prescribing in this country. Health Professionals at Enderby Medical Centre value the use of POC CRP testing as an asset. The test is quick, easy to use and reproducible. A tiny blood sample can provide a result in less than $6 \mathrm{~min}$ to help distinguish between potential viral and bacterial RTIs. The authors also found anecdotally that using the test allowed patients to be reassured about the illness and increased their understanding of antibiotic use in respiratory complaints. This hypothesis needs further evaluation and is currently research on-going in our unit.

The authors are certain that in primary care, POC CRP testing has a promising future. It can be used by nurse practitioners or health care assistants in GP surgeries or walk in centers or in urgent care setting. Research is still merited to elucidate whether this will have an impact on national rates of antibiotic use and this needs a larger call for randomized controlled trial of sufficient power. 


\section{Strengths and Limitations}

This is the first mixed methods case-control study in a United Kingdom General Practice setting to use POC CRP testing in conjunction with guidelines in the management of acute cough/ respiratory tract infection. The reference points for CRP testing have come from studies conducted within the secondary care sphere and mainly from pneumonia studies (ref). The added value of CRP might be different and could be lower when measured with a point of care test in general practice. Nonetheless, agreement between point of care test results and a conventional reference test has been shown to be good (refs).

The control group was essentially dependent on whether the system was accurate in picking up appropriate read codes for the diagnosis in the preceding year. As the clinicians involved in the study were unchanged through both time periods, this limitation has been curtailed and selection bias was kept to a minimum.

The authors also understand that given how common respiratory tract infections, the numbers in the intervention group were small but the demographics should make this study scale-able and applicable to the wider population.

To ensure appropriate use of the CRP test in the more clinically equivocal patients which are difficult to distinguish between viral and bacterial, a risk stratifying scoring system was used. Moreover such stratification avoided reliance on clinical examination findings such as chest findings which can be often is unreliable.

Samples were taken by one named nurse practitioner who was trained on how to calibrate and operate the CRP machine. This minimized errors and lack of data. Results were then interpreted by a qualified General Practitioner who then took appropriate action for that patient. Patients were then followed up within 2 weeks to ensure that safety was paramount.

\section{Conclusions}

POC CRP is a useful asset to have in a general practice and this study supports its use in conjunction with clinical findings in the management of ARTIs.

The test can be used by various health care professionals and improve confidence in decision making and reduce the use of antibiotic prescriptions.

\section{References}

1. Goossens H, Ferech M, Vander Stichele R, Elseviers M, ESAC Project Group (2005) Outpatient antibiotic use in Europe and association with resistance: a cross-national database study. Lancet 365: 579-5787.

2. Harbarth S, Samore MH (2005) Antimicrobial Resistance Determinants and Future Control. Emergency Inf Dis11: 795-801.

3. Kuyvenhoven MM, van Balen FA, Verheij TJ (2003) Outpatient antibiotic prescriptions from 1992 to 2001 in the Netherlands. J Antimicrob Chemother 52: 675-678

4. Linder JA, Singer DE, Strafford RS (2003) Association between antibiotics prescribing and visit duration in adults with upper respiratory tract infections. Clin Ther 25: 2419-2430.
5. Gonzales R, Anderer T, McCulloch CE, Maselli JH, Bloom FJ, et al. (2013) A cluster randomised trial decision support strategies for reducing antibiotic use in acute bronchitis. JAMA Intern Med 173: 267-273.

6. Butler CC, Hood K, Verheij T, Little P, Melbye H, et al. (2009) Variation in antibiotic prescribing and its impact on recovery in patients with acute cough in primary care: prospective study in 13 countries. BMJ 338: b2242.

7. Harrison PF, Lederberg J (1998) Antimicrobial resistance: issues and options. Workshop report. Forum on Emerging Infections, Division of Health Sciences Policy, Institute of Medicine. National Academy Press, Washington, D.C, USA. P: 1,39-41.

8. Little P, Dorward M, Warner G, Stephens K, Senior J, et al. (2004) Importance of patient pressure and perceived pressure and perceived medical need for investigations, referral, and prescribing in primary care: nested observational study. BMJ 328: 444-449.

9. Coenen S, Michiels B, Renard D, Denekens J, Van Royen P (2006) Antibiotic prescribing for acute cough: the effect of perceived patient demand. Br J Gen Pract 56: 183-190.

10. Jakobsen KA, Melbye H, Kelly MJ, Ceynowa C, Mölstad S, et al. (2010)Influence of CRP testing and clinical findings on antibiotic prescribing in adults presenting with acute cough in primary care. Scand J Prim Health Care 28: 229-236.

11. Gabay C, Kushner I (1999) Acute-phase proteins and other systemic responses to inflammation. N Engl J Med 340: 448-454.

12. Huang Y, Chen, Wu T, Wei X, Guo A (2013) Association between pointof-care CRP testing and antibiotic prescribing in respitatory tract infections: a systematic review and meta-analysis of primary care studies. Br J Gen Pract 63: 787-794.

13. Aabenhus R, Jensen JU, Jørgensen KJ, Hróbjartsson A, Bjerrum L (2014) Biomarkers as point-of-care tests to guide prescription of antibiotics in patients with acute respiratory infections in primary care. Cochrane Database Syst Rev 11: CD010130.

14. Little P, Stuart B, Francis N, Douglas E, Tonkin-Crine S, et al. (2013) Effects of internet-based training on antibiotic prescribing rates for acute respiratory-tract infections: A multinational, cluster, randomized, factional, controlled trial. Lancet 382: 1175-1182.

15. (2016) Winter pressure in accident and emergency departments Third Report of Session 2016. House of Commons Health Committee.

16. (2016) QuikRead go for C-reactive protein testing in primary care (mib78). Medtech innovation briefing. National Institute for Health and Care Excellence.

17. (2014) Pneumonia in adults: diagnosis and management (CG1291) National Institute for Health and Care Excellence.

18. van Vugt SF, Broekhuizen BD, Lammens C, Zuithoff NP, de Jong PA, et al (2013) Use of serum $C$ reactive protein and procalcitonin concentrations in addition to symptoms and signs to predict pneumonia in patients presenting to primary care with acute cough: diagnostic study. BMJ 346 : f2 2450 .

19. Cooke J, Butler C, Hopstaken R, Dryden MS, McNulty C, et al. (2015) Narrative review of primary care point-of-care testing (POCT) and antibacterial use in respiratory tract infection (RTI). BMJ Open Respir Res 2: e000086.

20. (2009) ECDC/EMEA Joint technical report. The bacterial challenge: Time to react. European Centre for Disease Prevention and Control.

21. Currie CJ, Berni E, Jenkins-Jones S, Poole CD, Ouwens M, Driessen S, et al. (2014) Antibiotic treatment failure in four common infections in UK primary care 1991-2012: Longitudinal analysis. Br Med J 349: g5493. 